\title{
Real-time measurement of ICD lead motion during stereotactic body radiotherapy of ventricular tachycardia
}

\author{
Lukas Knybel ${ }^{1}$, Jakub Cvek ${ }^{1}$, Radek Neuwirth ${ }^{2}$, Otakar Jiravsky ${ }^{3}$, Jan Hecko ${ }^{4}$, Marek Penhaker ${ }^{4}$, \\ Marek Sramko ${ }^{5}$, Josef Kautzner \\ ${ }^{1}$ Department of Oncology, University Hospital Ostrava, Ostrava, Czech Republic \\ ${ }^{2}$ MUNI- Masaryk University, Brno, Czech Republic \\ ${ }^{3}$ Department of Cardiology, Podlesi Hospital, Trinec, Czech Republic \\ ${ }^{4}$ VŠB-Technical University of Ostrava, Ostrava, Czech Republic \\ ${ }^{5}$ Institute for Clinical and Experimental Medicine, Prague, Czech Republic
}

\begin{abstract}
Background: Here we aimed to evaluate the respiratory and cardiac-induced motion of a ICD lead used as surrogate in the heart during stereotactic body radiotherapy (SBRT) of ventricular tachycardia (VT). Data provides insight regarding motion and motion variations during treatment.

Materials and methods: We analyzed the log files of surrogate motion during SBRT of ventricular tachycardia performed in 20 patients. Evaluated parameters included the ICD lead motion amplitudes; intrafraction amplitude variability; correlation error between the ICD lead and external markers; and margin expansion in the superior-inferior (SI), latero-lateral (LL), and anterior-posterior (AP) directions to cover $90 \%$ or $95 \%$ of all amplitudes.

Results: In the $\mathrm{SI}, \mathrm{LL}$, and AP directions, respectively, the mean motion amplitudes were $5.0 \pm 2.6,3.4$. \pm 1.9 , and $3.1 \pm 1.6$ $\mathrm{mm}$. The mean intrafraction amplitude variability was $2.6 \pm 0.9,1.9 \pm 1.3$, and $1.6 \pm 0.8 \mathrm{~mm}$ in the $\mathrm{SI}, \mathrm{LL}$, and AP directions, respectively. The margins required to cover $95 \%$ of ICD lead motion amplitudes were $9.5,6.7$, and $5.5 \mathrm{~mm}$ in the $\mathrm{SI}, \mathrm{LL}$, and $\mathrm{AP}$ directions, respectively. The mean correlation error was $2.2 \pm 0.9 \mathrm{~mm}$.

Conclusions: Data from online tracking indicated motion irregularities and correlation errors, necessitating an increased CTV-PTV margin of $3 \mathrm{~mm}$. In 35\% of cases, the motion variability exceeded $3 \mathrm{~mm}$ in one or more directions. We recommend verifying the correlation between CTV and surrogate individually for every patient, especially for targets with posterobasal localization where we observed the highest difference between the lead and CTV motion.
\end{abstract}

Key words: ventricular tachycardia; stereotactic body radiotherapy

Rep Pract Oncol Radiother 2021;26(1):128-137

\section{Introduction}

Patients with structural heart diseases carry substantial risks of scar-related ventricular tachycardia (VT) and arrhythmic sudden cardiac death. Over recent decades, the use of implantable cardioverter defibrillators (ICDs) has improved patient prog- noses following ventricular arrhythmia or cardiac arrest $[1,2]$, and in cases of severe systolic dysfunction and/or other risk factors [3, 4]. Main option is catheter ablation, which has substantial potential to destroy a focal source of arrhythmia and/or modify the arrhythmogenic substrate for reentrant arrhythmias, preventing or lessening their frequency [5,

Address for correspondence: Jakub Cvek, University Hospital Ostrava, Department of Oncology, 17. listopadu 1790, 70852 Ostrava, Czech Republic, tel: (+420) 59737 2125; e-mail: jakub.cvek@fno.cz 
6]. Catheter ablation shows better efficacy than escalation of antiarrhythmic therapy [7]. However, in $\sim 10 \%$ of cases, catheter ablation fails to abolish clinical VT [8].

Stereotactic body radiotherapy (SBRT) is primarily used for tumor treatment and exhibits high efficiency with minimal side effects. Recently, SBRT techniques with various forms of motion management have been investigated to destroy the critical part of the arrhythmogenic substrate in cases of failed catheter ablation [9-17].

When applying SBRT to targets in the thorax, motion management is critical. Any motion increases the risk of off-target delivery. As the indications for SBRT to treat tumors in the thorax expand, many methods of respiratory motion compensation have been developed. Precise dose delivery to the heart for SBRT of VT requires accounting for both respiratory motion and heartbeat. If respiratory motion is compensated with tracking, cardiac motion is compensated with an additional safety margin to target volume because cardiac-gated treatment is not available. Previous studies report that for tumors situated near the heart, the cardiac beat has a measurable impact on tumor movement [18-20]. The heart-induced tumor motion amplitude can be up to $2.6 \mathrm{~mm}$ [19], and left ventricle

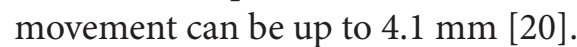

Real motion data from SBRT of VT could provide good insight into motion variations during treatment. In the present study, we performed the first analysis of implantable cardioverter defibrillator (ICD) lead motion used as surrogate during SBRT for ablation of VT. Our present data provides good insight regarding motion and motion variations during treatment, and may be useful for ITV delineation and treatment with conventional systems, or for safety margin delineation when tracking is used.

\section{Materials and methods}

This study included anonymized motion data from 20 patients (19 men, 1 woman) who underwent SBRT of VT. Data were collected with institutional review board (IRB) approval. Our treatment planning procedure has previously been described in detail $[10,11]$. Briefly, patients were treated with a single 25-Gy fraction using the CyberKnife system (Accuray, Inc., Sunnyvale, CA, USA) in con- junction with Synchrony tracking software that enabled real-time respiratory motion tracking. We used 3D maps from the electroanatomic mapping system (CARTO, Biosense-Webster, Israel) where the critical part of arrhythmogenic substrate was tagged. All tagged points were joined to one volume, uncertain points in the target area were included to the volume. For treatment planning, patients underwent expiratory and inspiratory breath-hold computed tomography (CT) scanning, and were coached not to perform maximum exhale and inhale. CT sets were used to verify the correlation between ICD lead position and the center of gravity (CoG) of the clinical target volume (CTV) during different phases of the breathing cycle. To account for heart contractions, we used electrocardiogram (ECG)-gated CT scans (in both systole and diastole) to delineate internal target volume (ITV). In the first 10 patients, to reduce potential toxicity, we provided no additional margin for planning target volume (PTV) delineation. At this time, there was only one reference relating to SBRT of VT in humans [21], and we were cautious about target expansion due to unknown side effects. After evaluating the efficiency and toxicity $[10,11]$ we added an additional 3-mm CTV-PTV margin in all subsequent cases. Dose distribution was calculated using Multiplan treatment planning system (Accuray, Inc., Sunnyvale, CA, USA). The required coverage was for $\geq 95 \%$ of the PTV to receive $25 \mathrm{~Gy}$, with the prescribed dose as close as possible to the $80 \%$ isodose line. Since all targets had an atypical (oblong) shape, the plans had a higher conformity index, which added an additional "dose margin" to the PTV. The end of the right ventricular septal ICD lead was used a the surrogate marker, and was continuously tracked with a robotic arm-mounted linear accelerator (LINAC). Live images were taken every $60 \mathrm{~s}$ during treatment, and the correlation model was continuously updated.

The Synchrony system does not allow compensating rotations during treatment if only a single marker is used. To minimize additional positional errors, we prepared an "alignment plan" for each patient, in which patient set-up was based on spine alignment (Xsight Spine). Using an alignment error of $<1 \mathrm{~mm}$ and $1^{\circ}$ ensured reproducibility of the patient's position at the start of treatment. After spine alignment, the patient was moved to the treatment center with a robotic couch. The first live image was 
acquired in the same breathing phase as in the digitally reconstructed radiographs (DRRs) generated from planning CT. We verified patient alignment in the treatment center using visible structures in the image, eg, ICD lead, chest wall, diaphragm, stainless steel wires in the patient's chest, and spine structures.

\section{Log file analysis}

We extracted the robot motion coordinates from the $\log$ files of the Synchrony motion tracking system. The technical aspects of the Synchrony system function and the clinical accuracy of this method have previously been described in detail [22-26]. The combined use of internal and external markers allows the robot to accurately follow target motion. Briefly, the Synchrony uses a kV-kV localizing system to detect the target or specific marker from two orthogonal X-ray images. The correlation model between the external LED markers on the patient's chest and the marker motion is constructed before the start of treatment, and is adapted during treatment with each new X-ray acquisition. The model can be rebuilt at any time during treatment if the correlation is worse than a user specified threshold. The motion of LEDs is tracked by optical surface tracking using a camera in the treatment room. Upon establishment of the correlation model, the treatment beam follows the internal movement via continuous measurement of external movement using the correspondence model, with compensation using the robot [27].

An in-house Matlab program (The MathWorks Inc., Natick, MA) was developed to determine motion amplitudes in the superior-inferior (SI), latero-lateral (LL), and anterior-posterior (AP) directions as local extremes in each breathing cycle. Based on amplitudes, this program generates information about margin expansion in the SI, LL, and AP directions to cover $90 \%$ or $95 \%$ of all amplitudes for a better description of motion variability.

\section{Motion analysis}

We evaluated motion amplitudes of marker (ICD lead) and intrafraction amplitude variability during treatment. Intrafraction amplitude variability was defined as the standard deviation (SD) of the ICD lead motion amplitude during one treatment fraction. Synchrony tracking accuracy depends on the correlation model and correlation error, ie, the dis- tance between the position given by the model and the position indicated by the X-ray. The correlation error was evaluated to enable quantification of an important source of inaccuracy. Synchrony uses 64 ms long X-ray acqusitions, which is sufficient time for static display of moving targets, and the correlation model is mostly based on linear function. It is assumed that heartbeat can randomly affect the marker position in different parts of the breathing cycle and, therefore, the correlation error between an external marker on the patient's chest and the ICD lead can increase. Therefore, the data can contain combined breathing and cardiac motion and couldn't be extracted separately.

\section{Tracking geometry}

Tracking accuracy can be affected by number of markers being tracked and the distance between the target and imaging center [28]. We recorded the distance between the end of the ICD lead (imaging center) and the CoG of the CTV as the magnitude of a vector between two points in 3D. Targets were divided into four groups based on location in the heart: anterolateral (AL), inferolateral (IL), inferior (I), and posterobasal (P) (Tab. 1). Figure 1 presents two examples of the relationship between the ICD lead and CTV.

\section{Fiducial marker definition}

In each case, the first correlation model had to be approved by the physician and physicist, to confirm tracking on the appropriate part of the electrode throughout the whole breathing cycle. Figure 2 shows the difficult part of tracking, during which it is necessary to carefully check the part of the electrode being tracked. The tracking point of the electrode is approximately $3-\mathrm{mm}$ wide (B and D). Synchrony tracking system automatically detects the tracking point; some cases required manual correction in comparison with the exact position on DRRs (set during treatment planning). We set the criterion to start/continue treatment only when the actual correlation error was $<3 \mathrm{~mm}$.

\section{Statistical analysis}

Statistical analysis was performed using the STATISTICA 10 software (StatSoft, Tulsa, OK). All quantitative data were expressed as mean and SD. Box-plots or histograms were constructed for visual comparison of the samples. 
Table 1. Distance between the end of the ICD lead (imaging center) and the clinical target volume center of gravity, target localization, conformity index, mean motion amplitude, and standard deviation (intrafraction motion variability) for all cases

\begin{tabular}{|c|c|c|c|c|c|c|c|c|c|}
\hline \multirow{2}{*}{ Patient } & \multirow{2}{*}{ Distance $[\mathrm{mm}]$} & \multirow{2}{*}{$\begin{array}{c}\text { Target } \\
\text { localization }\end{array}$} & \multirow{2}{*}{$\mathrm{Cl}^{*}$} & \multicolumn{3}{|c|}{ Mean motion amplitude [mm] } & \multicolumn{3}{|c|}{ Standard deviation [mm] } \\
\hline & & & & SI & LL & AP & SI & LL & AP \\
\hline 1 & 65.9 & AL & 1.27 & 4.7 & 5.9 & 3.1 & 1.8 & 3.5 & 2.0 \\
\hline 2 & 78.4 & $P$ & 1.67 & 4.6 & 3.3 & 3.6 & 2.8 & 2.1 & 2.1 \\
\hline 3 & 79.6 & I & 1.77 & 5.3 & 3.2 & 3.0 & 1.9 & 1.4 & 1.3 \\
\hline 4 & 88.5 & $P$ & 1.48 & 6.9 & 2.0 & 2.8 & 3.9 & 0.2 & 0.8 \\
\hline 5 & 69.8 & IL & 1.78 & 4.1 & 4.2 & 2.8 & 3.0 & 4.2 & 1.4 \\
\hline 6 & 56.3 & 1 & 1.28 & 4.3 & 2.2 & 2.6 & 2.3 & 0.5 & 0.9 \\
\hline 7 & 70.6 & $P$ & 1.58 & 3.9 & 2.7 & 3.2 & 1.5 & 1.0 & 1.2 \\
\hline 8 & 56.2 & I & 1.21 & 7.0 & 3.3 & 4.1 & 2.3 & 1.5 & 2.3 \\
\hline 9 & 68.9 & $P$ & 1.28 & 4.5 & 3.0 & 3.2 & 3.1 & 1.3 & 1.5 \\
\hline 10 & 36.4 & $A L$ & 1.24 & 6.3 & 3.0 & 2.1 & 4.6 & 2.4 & 4.4 \\
\hline 11 & 60.1 & I & 1.20 & 5.1 & 2.2 & 2.9 & 2.1 & 0.5 & 1.1 \\
\hline 12 & 73.4 & I & 1.15 & 4.9 & 2.8 & 2.9 & 1.5 & 0.9 & 0.8 \\
\hline 13 & 62.1 & IL & 1.29 & 4.8 & 2.7 & 2.7 & 2.4 & 1.4 & 1.2 \\
\hline 14 & 33.1 & IL & 1.24 & 5.8 & 2.1 & 4.9 & 2.0 & 0.4 & 1.6 \\
\hline 15 & 69.0 & 1 & 1.45 & 7.3 & 3.9 & 3.9 & 4.8 & 3.7 & 2.9 \\
\hline 16 & 28.6 & $\mathrm{AL}$ & 1.19 & 3.9 & 3.2 & 3.5 & 2.0 & 1.6 & 1.6 \\
\hline 17 & 96.3 & $P$ & 1.21 & 5.3 & 6.8 & 3.6 & 2.6 & 4.9 & 1.4 \\
\hline 18 & 87.3 & 1 & 1.31 & 3.2 & 4.6 & 2.8 & 2.0 & 2.7 & 1.1 \\
\hline 19 & 67.1 & IL & 1.15 & 3.4 & 3.5 & 2.6 & 1.8 & 2.1 & 1.1 \\
\hline 20 & 24.4 & $\mathrm{AL}$ & 1.38 & 5.3 & 2.7 & 2.5 & 2.5 & 1.6 & 1.8 \\
\hline Mean & 63.6 & & 1.4 & 5.0 & 3.4 & 3.1 & 2.6 & 1.9 & 1.6 \\
\hline
\end{tabular}

*Conformality index; AL — anterolateral; IL — inferolateral; I — inferior; P — posterobasal; Highlighted values indicate dominant mean motion in a direction other than $\mathrm{SI}$ and intrafraction motion variability of $>3 \mathrm{~mm}$ in any direction

\section{Results}

We analyzed a total of 20 fractions, with a mean duration of $63 \pm 12.3$ minutes. In the SI, LL, and AP directions, respectively, the mean motion amplitudes were $5.0 \pm 2.6 \mathrm{~mm}, 3.4 . \pm 1.9 \mathrm{~mm}$, and $3.1 \pm 1.6 \mathrm{~mm}$ (Tab. 1); and the mean maximum motion amplitudes detected during treatment were $20.8 \pm 7.5 \mathrm{~mm}, 13.7 \pm 7.5 \mathrm{~mm}$, and $12.4 \pm 5.0 \mathrm{~mm}$. All maximum values were detected during coughing or sudden deep inspiration, when the treatment was stopped due to excessive motion of the external LED markers. Box plots in Figure 3 show the mean motion amplitude data in the SI, LL and AP directions. We compared the coordinates of the ICD lead and CTV CoG from expirium and inspirium CT. In 17 cases, the lead and CTV CoG motion differed by $\leq 1.5 \mathrm{~mm}$ in the SI, LL, or AP direction.
In three cases $(2,4$, and 7$)$ with a posterobasally localized target, the difference was up to $3 \mathrm{~mm}$ in one direction.

\section{Intrafraction amplitude variability}

The mean intrafraction amplitude variability was $2.6 \pm 0.9 \mathrm{~mm}, 1.9 \pm 1.3 \mathrm{~mm}$ and $1.6 \pm 0.8 \mathrm{~mm}$ in the SI, LL, and AP directions, respectively. Variability of $>3 \mathrm{~mm}$ in one or more directions was reported in seven cases (Tab. 1).

\section{Margins covering 90/95\% of the lead motion}

To better describe the motion and motion variability of the lead, we generated the margins that were necessary to cover $90 \%$ or $95 \%$ of the lead motion amplitudes in the SI, LL, and AP directions (Fig. 4). 


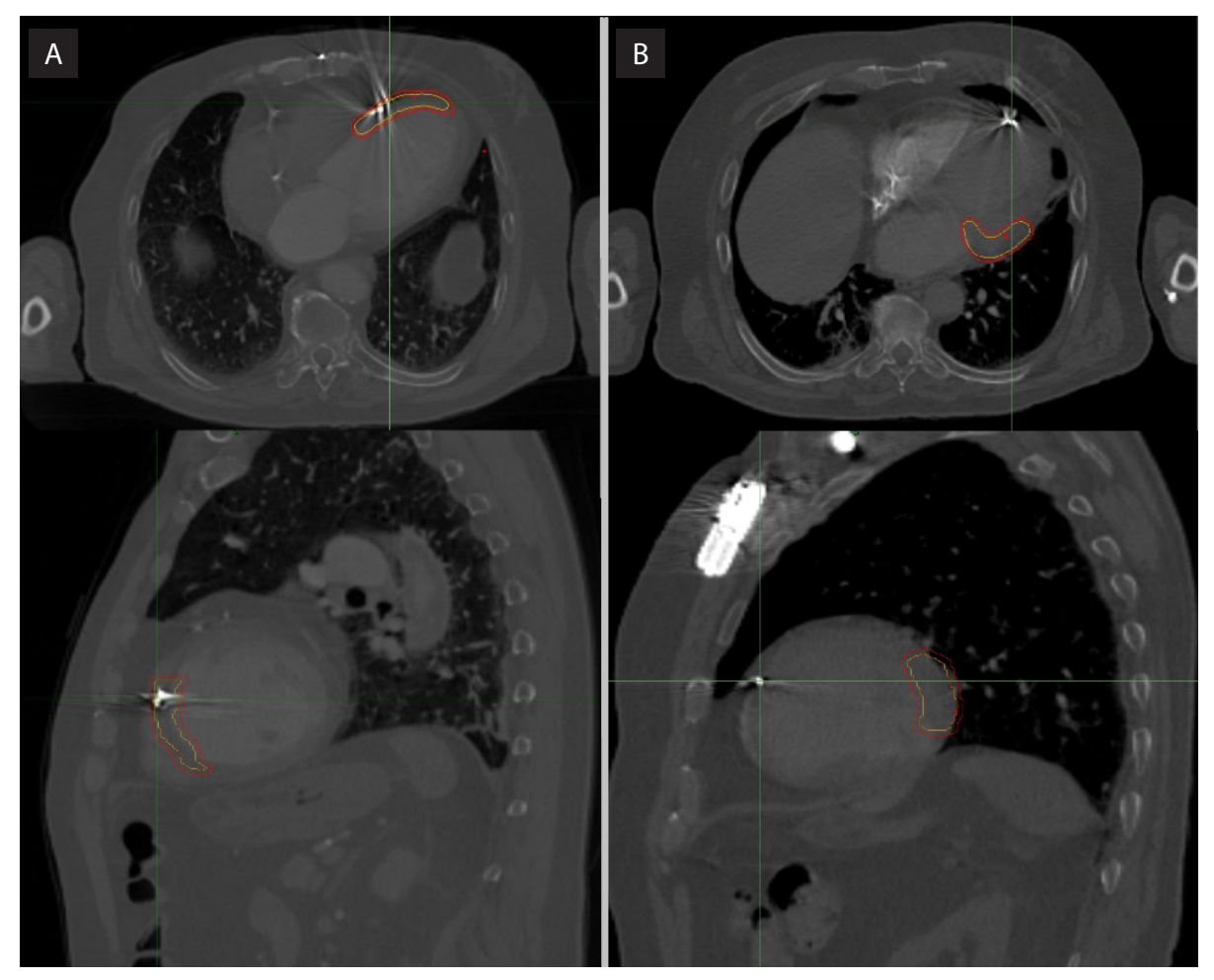

Figure 1. Examples of position of the implantable cardioverter defibrillator (ICD) lead and target volume. A. A case in which the lead was in contact with the clinical target volume (CTV) in the transversal and sagittal planes. B. A case with the highest distance between the ICD lead and CTV in the transversal and sagittal planes (PTV $=$ CTV $+3 \mathrm{~mm}$ )
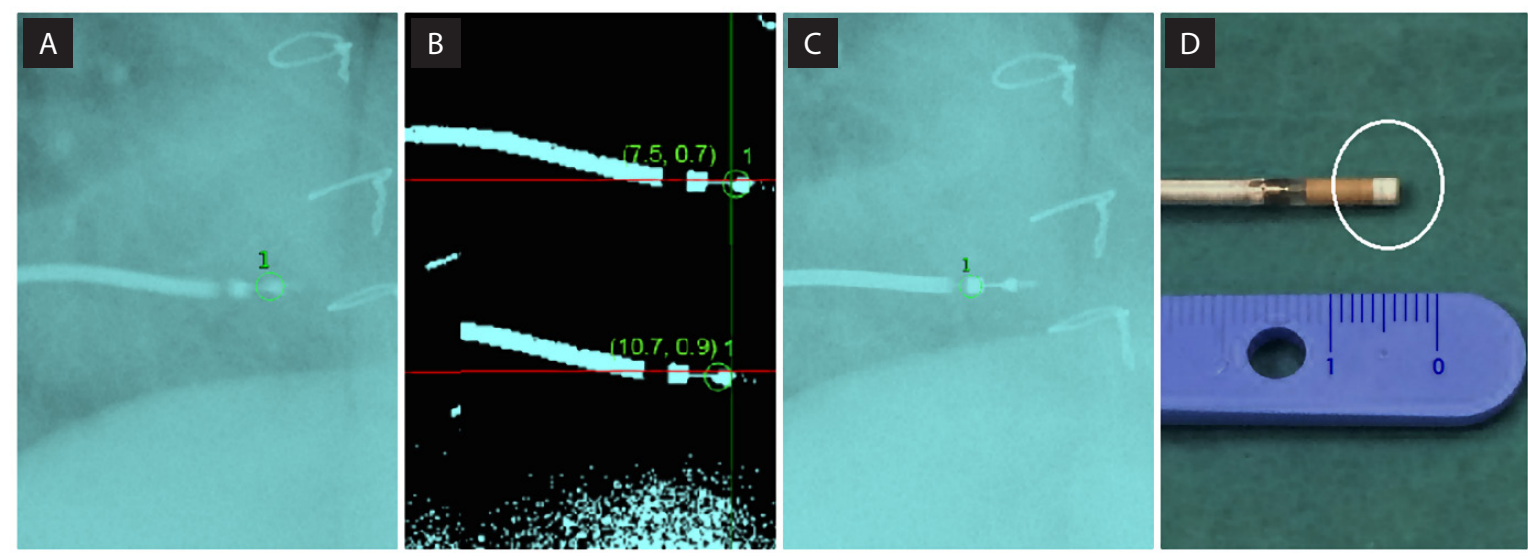

Figure 2. The part of the electrode being used for tracking. A. A live X-ray image acquired during treatment, with the end of the ICD lead correctly identified according to the defined position during planning. B. The source of possible tracking uncertainty, with enhanced contrast. Crosshairs indicate the left and right ends of the electrode with visible LL coordinates [mm]. Both ends are incorrectly identified parts of the lead relative to the DRRs, which could be tracked under certain assumptions. The diameter is approximately $3 \mathrm{~mm}$. C. An example of incorrectly identified end of the ICD lead which require manual correction. D. The end of the electrode (white circle) before implantation, relative to a metric ruler [cm]. Box-plots representing the mean motion amplitude in the the superior-inferior (SI), latero-lateral (LL), and anterior-posterior (AP) directions

\section{Correlation error}

Since the correlation error was consistently over $1 \mathrm{~mm}$, we cannot exclude the mutual influence of patient alignment and heartbeat. In cases 10, 14, 16, and 20, the lead was in direct contact with part of the CTV. Although we set the criterion to start/continue treatment only when the actual correlation error was $<3 \mathrm{~mm}$, several values above $3 \mathrm{~mm}$ were re- 


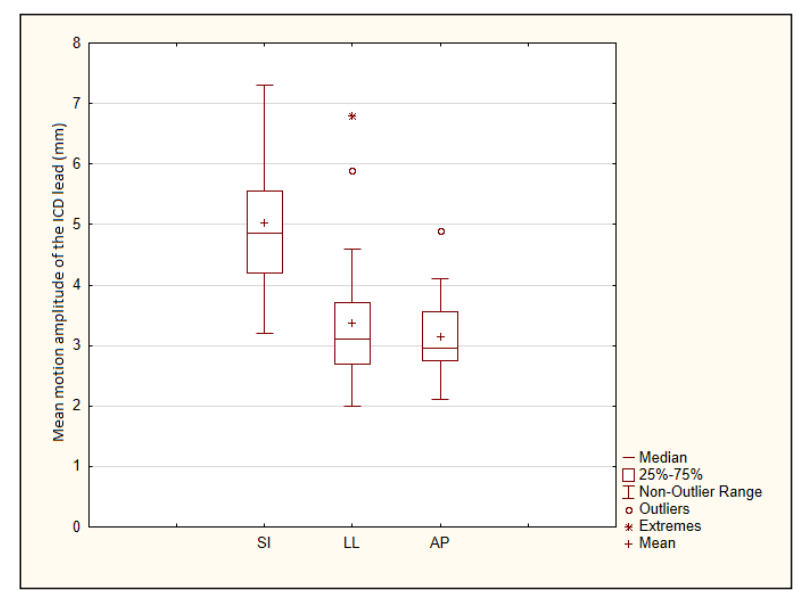

Figure 3. Box-plots representing the mean motion amplitude in the superior-inferior (SI), latero-lateral (LL), and anterior-posterior (AP) directions

corded during treatment. When a correlation error of $>3 \mathrm{~mm}$ was detected in two consecutive images, the patient was realigned. On average, we had to establish 5 (SD 3.3) correlation models per treatment, and the mean correlation error was $2.2 \pm 0.9 \mathrm{~mm}$.

\section{Discussion}

Previous studies that have reported outcomes of SBRT for VT ablation can be divided into two treatment approaches based on the utilized technical equipment and motion management. One approach includes the use of the CyberKnife system with online tracking in which the end of the ICD lead is used as a tracking marker [10, $11,16]$. The other approach involves internal target volume (ITV) treatment with conventional LINAC, and treatment delivery with respiratory-gated 4DCT for target definition [13-15]. For both approaches, the overall treatment accuracy remains unclear. In our present study, we aimed to describe the ICD lead motion during SBRT of VT. To our knowledge, this was the first work to describe this motion based on online tracking during SBRT of VT.

Our results in the SI, LL, and AP directions, respectively, showed that the mean motion amplitudes were $5.0 \pm 2.6 \mathrm{~mm}, 3.4 \pm 1.9 \mathrm{~mm}$, and $3.1 \pm 1.6 \mathrm{~mm}$; and the mean maximum motion amplitudes detected during treatment were $20.8 \pm 7.5 \mathrm{~mm}, 13.7 \pm 7.5 \mathrm{~mm}$, and $12.4 \pm 5.0$ $\mathrm{mm}$. The defective part of the heart was treated, in which cardiac motion is expected to be lower compared to healthy heart. The present data described the combination of cardiac and breathing motion. Knutson et al. [15] reported data from $4 \mathrm{D}$-CT with bridge abdominal compression in 16

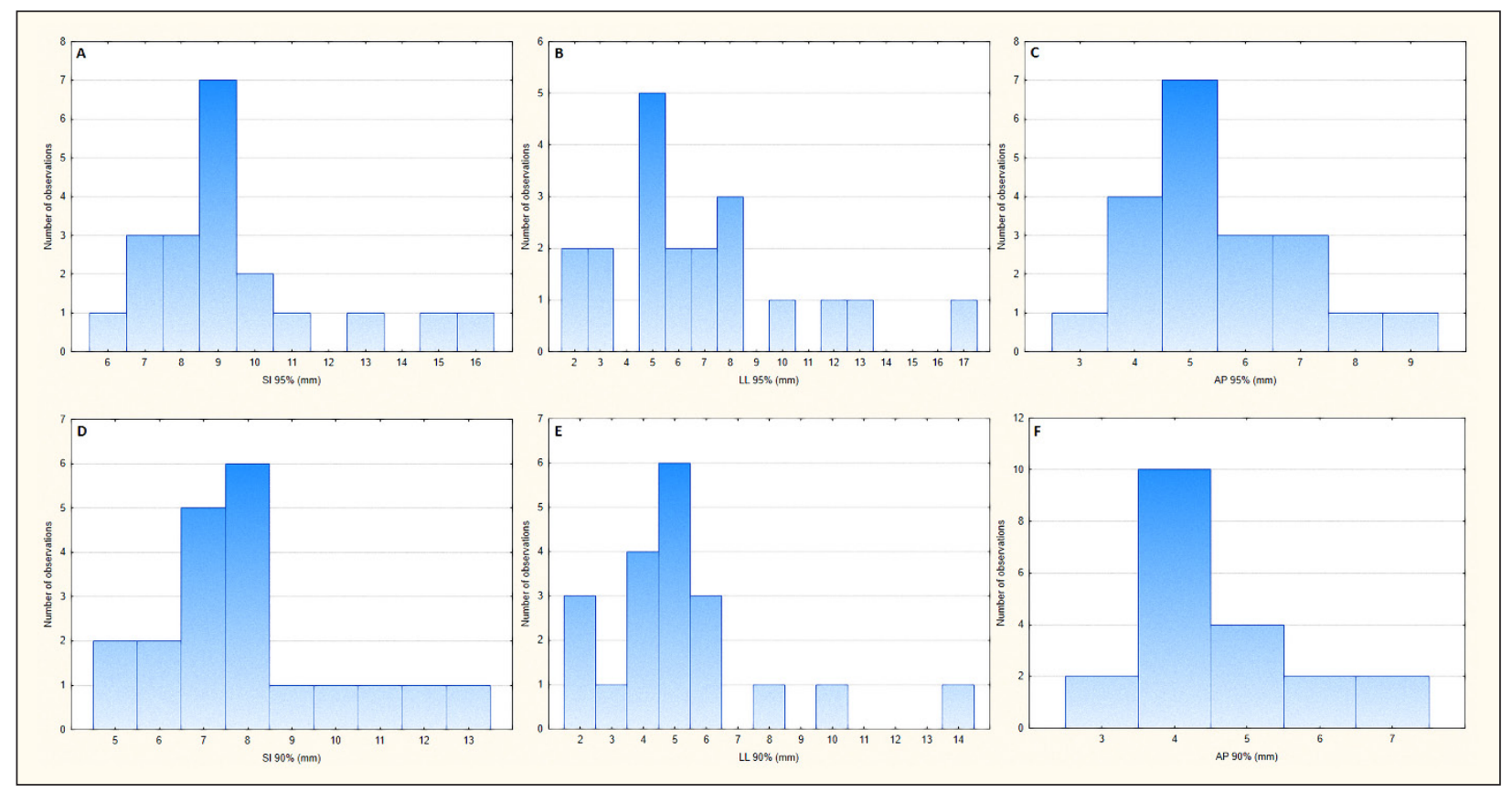

Figure 4. The margins [mm] covering $90 / 95 \%$ of the lead motion. The margins [mm] necessary to cover $95 \%$ (A-C) and $90 \%$ (D-F) of the lead motion amplitudes, and the numbers of observations, in the the superior-inferior (SI), latero-lateral (LL), and anterior-posterior (AP) directions 
patients treated with conventional LINAC for VT. The median-maximum distance from the GTV to the ITV was $4.4 \mathrm{~mm}$ (minimum: $3 \mathrm{~mm}$, maximum: $11.3 \mathrm{~mm}$ ) in the axial plane, $4.65 \mathrm{~mm}(1.55$, $12)$ in the coronal plane, and $3 \mathrm{~mm}(1,7.2)$ in the sagittal plane. These results are comparable to our data from free-breathing treatment; however, we observed higher maximal motion amplitudes during treatment without the use of abdominal compression. Tong et al. [20] used the breath-hold technique to quantify variations in the heart, pericardium, and left ventricular myocardium (LVM) caused by cardiac movement. Results obtained with the breath-hold technique should not be affected by breathing motion. LVM motion validation provides useful information for SBRT treatments. However, in their study, the LVM was delineated without the interventricular septum, which is where the end of the lead was in our present study. They reported that the largest displacement was $4.1 \pm 2.8 \mathrm{~mm}$, which occurred in the LVM along the AP axis. However, it is difficult to compare results obtained using the breath-hold technique to those acquired with free breathing.

Chen et al. [19] evaluated cardiac-induced lung tumor motion and found that the heart-induced motion amplitude ranged from 0.2 to $2.6 \mathrm{~mm}$. Based on fluoroscopy, they reported average lung impacts of $3.6 \pm 3.55 \mathrm{~mm}$ in the SI direction and $1.42 \pm 1.09 \mathrm{~mm}$ in the LL direction, and average heart impacts of $0.82 \pm 0.65$ and $0.52 \pm 0.31 \mathrm{~mm}$, respectively. Compared to our present findings, they reported a similar average motion in the SI direction, and an average motion in the LL direction that was almost twice as low. However, it is notable that their results describe the impact on tumors near the heart rather than on the heart itself.

Seppenwoolde et al. [18] measured tumor motion in the lung due to heartbeat. Their finding that is most relevant to SBRT of VT is that the heartbeat-induced motion was the greatest in the LL direction (range, $1-4 \mathrm{~mm}$ ). In our previous study [26], we observed intrafraction amplitude variability of $>3 \mathrm{~mm}$ in cases where the tumo $r$ was in contact with another structure, such as the heart or diaphragm. In our present study, $25 \%$ of cases had greater motion in the LL direction compared the SI direction. Ross et al. [29] analyzed the movement of intrathoracic neoplasms using ultrafast computed tomography, and reported that lateral movement was most pronounced for tumors located adjacent to the aorta or heart. This appeared to result from aortic pulsation, as well as from cardiac contraction.

Although it is possible to perform tracking using a single marker, there remains some uncertainty. Such tracking is limited to linear chasing and does not track rotations [30]. When using a single marker, there is an implicit assumption that the location of this fiducial marker represents the median/average of the target volume [31]. Seppenwoolde et al. [33] evaluated how tumor prediction errors were related to the distance between the surrogate (single marker) and tumor during liver SBRT. The estimated random and systematic prediction errors were $\leq 2.8 \mathrm{~mm}$ in any direction for distances up to $100 \mathrm{~mm}$. Christ et al. [30] investigated the impact of rotational deviations on single-fiducial-based respiratory tracking, with the hypothesis being that the yaw (clockwise) rotation would increase the tracking uncertainty. They reported that an angle of $\geq 40^{\circ}$ caused treatment inaccuracy of $>1 \mathrm{~mm}$ with single fiducial inside the target. In a related study, Fuchs et al. [32] investigated left ventricular (LV) contraction and relaxation in patients with chronic heart failure (CHF), and reported that LV rotation in these patients was reduced at both the base $\left(-3.4 \pm 2^{\circ}, \mathrm{p}<.01\right)$ and the apex $\left(+0.9 \pm 3^{\circ}\right.$, $\mathrm{p}<.05)$. Similarly, CHF patients showed a reduced regional ejection fraction (REF) at both the base and apex. This fact can help to reduce the inaccuracy of SBRT of VT. All of our evaluated patients had an ejection fraction of $<35 \%$ and severe cardiac insufficiency.

The distance between the target and marker is also important. We recorded the distance between the end of the ICD lead and the CoG of the CTV as the magnitude of a vector between two points in 3D (Tab. 1). When the marker is placed outside of the target, there is an assumption that the marker motion corresponds to the motion of the target being treated. This must be verified during the planning process and included in the safety margin. We did not increase the safety margin more due to the proximity of the organ at risk and the higher conformity index caused by the atypical target shape. To minimize the impact of the mentioned distance, patients were coached to breathe normally. Braunstein et al. [28] compared local recurrence (LR) of lung tumors treated with direct tumor imaging 
vs with an indirect spine tracking method that is similarly problematic to the tracking of an ICD lead outside of the target. Their results showed increased LR in cases where spine-based tumor tracking was performed with an imaging center $>4$ $\mathrm{cm}$ from the target. Notably, the target in this study was not a tumor. While the aim of ablative tumor irradiation is to destroy every clonogen cell, it remains unclear whether the same goal is necessary for scar-related VT.

Online tracking data can elucidate target motion during treatment of VT, but there are several limitations. The target trajectory is indirectly estimated using the correlation model of the Synchrony system compared to directly obtained datasets. With a known correlation error, we can quantify the possible treatment inaccuracy. We found that our correlation error was consistently higher compared to lung treatments [26], which may be due to several possible causes: (1) the patient's rotation caused rotation of the ICD lead, making tracking more difficult (patients were realigned when a correlation error of $>3 \mathrm{~mm}$ was detected in two consecutive images; (2) longer treatment can be related to less stable patient alignment; (3) if the end of the ICD lead was oriented perpendicularly to the imaging system, some information about lead orientation is lost; (4) if the patient was breathing very deeply, the end parts of the breathing cycle yielded the highest correlation errors ( $>4 \mathrm{~mm}$ ); and (5) the correlation between an external marker on the patient's chest and the ICD lead can be affected by heartbeat (accordingly, Teo et al. [34] reported potential softening of the dose gradient changes due to cardiac motion). To maintain sufficient coverage of the target, we created patient-specific ITV from ECG-gated CT scans to account for heart contractions.

\section{Conclusion}

Our present data from online tracking indicated motion irregularities and correlation errors that necessitated an increased CTV-PTV margin of $3 \mathrm{~mm}$. Motion variability of $>3 \mathrm{~mm}$ in one or more directions was detected in $35 \%$ of cases. The ICD lead motion correlated with the CTV CoG motion with translational difference $\leq 1.5 \mathrm{~mm}$. In three cases with a posterobasally localized target, the difference was up to $3 \mathrm{~mm}$ in one direction. We recommend a verification of the correlation between CTV and surrogate for every patient, especially for a target localized posterobasal in the heart where we observed the highest difference between the lead and PTV motion. Patient specific margins should be considered in the future.

\section{Conflict of interest}

The authors have no affiliation with any organization with a direct or indirect financial interest in the subject matter discussed in the manuscript. Authors have no financial interests related to the material in the manuscript.

L.K., J.C., R.N., O.J., J.H., M.P., M.S. have nothing to disclose. J.K. reports personal fees and other from Biosense Webster, personal fees and other from Bostron Scientific, personal fees and other from Epix , personal fees from LIva Nova (MicroPort), personal fees and other from Medtronic, from null, personal fees and other from Abbott (SJM), personal fees and other from Daiichi Sankyo, personal fees from Boehringer Ingelheim, personal fees from Bayer, personal fees from Pfizer, personal fees from MSD, personal fees and other from Affera, outside the submitted work.

\section{Funding}

Funding information is not available.

\section{Authors' contribution}

All authors have participated in (a) conception and design, or analysis and interpretation of the data; (b) drafting the article or revising it critically for important intellectual content; and (c) approval of the final version.

\section{References}

1. Antiarrhythmics versus Implantable Defibrillators (AVID) Investigators. A comparison of antiarrhythmic-drug therapy with implantable defibrillators in patients resuscitated from near-fatal ventricular arrhythmias. N Engl J Med. 1997; 337(22): 1576-1583, doi: 10.1056/ NEJM199711273372202, indexed in Pubmed: 9411221.

2. Connolly S. Meta-analysis of the implantable cardioverter defibrillator secondary prevention trials. AVID, CASH and CIDS studies. Antiarrhythmics vs Implantable Defibrillator study. Cardiac Arrest Study Hamburg . Canadian Implantable Defibrillator Study. Eur Heart J. 2000; 21 (24): 2071-2078, doi: 10.1053/euhj.2000.2476, indexed in Pubmed: 11102258.

3. Moss AJ, Zareba W, Hall WJ, et al. Multicenter Automatic Defibrillator Implantation Trial II Investigators. Prophylactic implantation of a defibrillator in patients with myocardial infarction and reduced ejection fraction. N Engl J Med. 
2002; 346(12): 877-883, doi: 10.1056/NEJMoa013474, indexed in Pubmed: 11907286.

4. Nanthakumar K, Epstein AE, Kay GN, et al. Prophylactic implantable cardioverter-defibrillator therapy in patients with left ventricular systolic dysfunction: a pooled analysis of 10 primary prevention trials. J Am Coll Cardiol. 2004; 44(11): 2166-2172, doi: 10.1016/j.jacc.2004.08.054, indexed in Pubmed: 15582314.

5. Mallidi J, Nadkarni GN, Berger RD, et al. Meta-analysis of catheter ablation as an adjunct to medical therapy for treatment of ventricular tachycardia in patients with structural heart disease. Heart Rhythm. 2011; 8(4): 503-510, doi: 10.1016/j.hrthm.2010.12.015, indexed in Pubmed: 21147263.

6. Marchlinski FE, Haffajee $\mathrm{Cl}$, Beshai JF, et al. Long-Term Success of Irrigated Radiofrequency Catheter Ablation of Sustained Ventricular Tachycardia: Post-Approval THERMOCOOL VT Trial. J Am Coll Cardiol. 2016; 67(6): 674-683, doi: 10.1016/j.jacc.2015.11.041, indexed in Pubmed: 26868693.

7. Sapp JL, Wells GA, Parkash R, et al. VentricularTachycardia Ablation versus Escalation of Antiarrhythmic Drugs. N Engl J Med. 2016; 375(2): 111-121, doi: 10.1056/NEJMoa1513614, indexed in Pubmed: 27149033.

8. Tokuda M, Kojodjojo P, Tung S, et al. Acute failure of catheter ablation for ventricular tachycardia due to structural heart disease: causes and significance. J Am Heart Assoc. 2013; 2(3): e000072, doi: 10.1161/JAHA.113.000072, indexed in Pubmed: 23727700.

9. Loo BW, Soltys SG, Wang L, et al. Stereotactic ablative radiotherapy for the treatment of refractory cardiac ventricular arrhythmia. Circ Arrhythm Electrophysiol. 2015; 8(3): 748-750, doi: 10.1161/CIRCEP.115.002765, indexed in Pubmed: 26082532.

10. Loo BW, Soltys SG, Wang L, et al. Stereotactic ablative radiotherapy for the treatment of refractory cardiac ventricular arrhythmia. Circ Arrhythm Electrophysiol. 2015; 8(3): 748-750, doi: 10.1161/CIRCEP.115.002765, indexed in Pubmed: 26082532.

11. Cvek J, Neuwirth R, Knybel L, et al. Cardiac radiosurgery for malignant ventricular tachycardia. Cureus. 2014; 6(7), doi: e190. doi:10.7759/cureus.190.

12. Gianni C, Mohanty S, Trivedi C, et al. Alternative Approaches for Ablation of Resistant Ventricular Tachycardia. Card Electrophysiol Clin. 2017; 9(1): 93-98, doi: 10.1016/j. ccep.2016.10.006, indexed in Pubmed: 28167089.

13. Cuculich PS, Schill MR, Kashani R, et al. Noninvasive Cardiac Radiation for Ablation of Ventricular Tachycardia. N Engl J Med. 2017; 377(24): 2325-2336, doi: 10.1056/ NEJMoa1613773, indexed in Pubmed: 29236642.

14. Robinson CG, Samson PP, Moore KM, et al. Phase I/IITrial of Electrophysiology-Guided Noninvasive Cardiac Radioablation for VentricularTachycardia. Circulation. 2019; 139(3): 313-321, doi: 10.1161/CIRCULATIONAHA.118.038261, indexed in Pubmed: 30586734.

15. Knutson NC, Samson PP, Hugo GD, et al. Radiation Therapy Workflow and Dosimetric Analysis from a Phase 1/2 Trial of Noninvasive Cardiac Radioablation for Ventricular Tachycardia. Int J Radiat Oncol Biol Phys. 2019; 104(5): 1114-1123, doi: 10.1016/j.ijrobp.2019.04.005, indexed in Pubmed: 31002942.

16. Jumeau R, Ozsahin M, Schwitter J, et al. Rescue procedure for an electrical storm using robotic non-invasive cardiac radio-ablation. Radiother Oncol. 2018; 128(2): 189-191, doi: 10.1016/j.radonc.2018.04.025, indexed in Pubmed: 29753550.

17. Haskova J, Peichl P, Pirk J, et al. Stereotactic radiosurgery as a treatment for recurrent ventricular tachycardia associated with cardiac fibroma. HeartRhythm Case Rep. 2019; 5(1): 44-47, doi: 10.1016/j.hrcr.2018.10.007, indexed in Pubmed: 30693205.

18. Seppenwoolde $Y$, Shirato H, Kitamura K, et al. Precise and real-time measurement of 3D tumor motion in lung due to breathing and heartbeat, measured during radiotherapy. Int J Radiat Oncol Biol Phys. 2002; 53(4): 822-834, doi: 10.1016/ s0360-3016(02)02803-1, indexed in Pubmed: 12095547.

19. Chen T, Qin S, Xu X, et al. Frequency filtering based analysis on the cardiac induced lung tumor motion and its impact on the radiotherapy management. Radiother Oncol. 2014; 112(3): 365-370, doi: 10.1016/j.radonc.2014.08.007, indexed in Pubmed: 25236714.

20. Tong Y, Yin Y, Lu J, et al. Quantification of heart, pericardium, and left ventricular myocardium movements during the cardiac cycle for thoracic tumor radiotherapy. Onco Targets Ther. 2018; 11: 547-554, doi: 10.2147/OTT. S155680, indexed in Pubmed: 29416355.

21. Zei PC, Soltys SG, Loo B. First-in-man treatment of arrhythmia (ventricular tachycardia) using stereotactic radiosurgery. Heart Rhythm. 2013; 10: 1-554.

22. Murphy MJ. Fiducial-based targeting accuracy for external-beam radiotherapy. Med Phys. 2002; 29(3): 334-344, doi: 10.1118/1.1448823, indexed in Pubmed: 11929016.

23. Hoogeman M, Prévost JB, Nuyttens J, et al. Clinical accuracy of the respiratory tumor tracking system of the cyberknife: assessment by analysis of log files. Int J Radiat Oncol Biol Phys. 2009; 74(1): 297-303, doi: 10.1016/j. ijrobp.2008.12.041, indexed in Pubmed: 19362249.

24. Pepin EW, Wu H, Zhang Y, et al. Correlation and prediction uncertainties in the cyberknife synchrony respiratory tracking system. Med Phys. 2011; 38(7): 4036-4044, doi: 10.1118/1.3596527, indexed in Pubmed: 21859002.

25. Dieterich S, Green O, Booth J. SBRT targets that move with respiration. Phys Med. 2018; 56: 19-24, doi: 10.1016/j. ejmp.2018.10.021, indexed in Pubmed: 30527085.

26. Knybel L, Cvek J, Molenda L, et al. Analysis of Lung Tumor Motion in a Large Sample: Patterns and Factors Influencing Precise Delineation of Internal Target Volume. Int J Radiat Oncol Biol Phys. 2016; 96(4): 751-758, doi: 10.1016/j. ijrobp.2016.08.008, indexed in Pubmed: 27788948.

27. Seppenwoolde Y, Berbeco RI, Nishioka S, et al. Accuracy of tumor motion compensation algorithm from a robotic respiratory tracking system: a simulation study. Med Phys. 2007; 34(7): 2774-2784, doi: 10.1118/1.2739811, indexed in Pubmed: 17821984.

28. Braunstein SE, Descovich M, Johnson JA, et al. Effect of Stereotactic Tracking Method on Local Control in Early Stage Non-Small Cell Lung Cancer (NSCLC). Int J Radiat Oncol Biol Phys. 2014; 90(1): S19, doi: 10.1016/j. ijrobp.2014.05.109.

29. Ross C, Hussey D, Pennington E, et al. Analysis of movement of intrathoracic neoplasms using ultrafast computerized tomography. Int J Rad Oncol Biol Phys. 1990; 18(3): 671-677, doi: 10.1016/0360-3016(90)90076-v, indexed in Pubmed: 2318701.

30. Christ Z, Shang CY, Gibbard G, et al. Impact of Rotational Deviations on a Single Fiducial Based Respiratory 
Tracking. Int J Radiat Oncol Biol Phys. 2017; 99(2): E650, doi: 10.1016/j.ijrobp.2017.06.2171.

31. Wu X, Dieterich S, Orton CG. Point/counterpoint. Only a single implanted marker is needed for tracking lung cancers for IGRT. Med Phys. 2009; 36(11): 4845-4847, doi: 10.1118/1.3218765, indexed in Pubmed: 19994491.

32. Fuchs $E$, Müller MF, Oswald H, et al. Cardiac rotation and relaxation in patients with chronic heart failure. Eur J Heart Fail. 2004; 6(6): 715-722, doi: 10.1016/j.ejheart.2003.12.018, indexed in Pubmed: 15542406.
33. Seppenwoolde $Y$, Wunderink W, Wunderink-van Veen $S R$, et al. Treatment precision of image-guided liver SBRT using implanted fiducial markers depends on marker-tumour distance. Phys Med Biol. 2011; 56(17): 5445-5468, doi: 10.1088/0031-9155/56/17/001, indexed in Pubmed: 21813963.

34. Teo BK, Dieterich S, Blanck O, et al. SU-FF-T-559: Effect of Cardiac Motion On the Cyberknife Synchrony Tracking System for Radiosurgical Cardiac Ablation. Med Phys. 2009; 36(6Part17): 2653-2653, doi: 10.1118/1.3182057. 